The Astrophysical Journal, 385:255-264, 1992 January 20

(C) 1992. The American Astronomical Society. All rights reserved. Printed in U.S.A.

\title{
THE SPECTRA OF IC 418 AND IC 5117 FROM 1.5 TO 1.75 MICRONS
}

\author{
C. Y. Zhang AND SUN KwoK \\ Department of Physics and Astronomy, The University of Calgary, Calgary, Alberta, Canada T2N 1N4 \\ Received 1991 February 25 ; accepted 1991 July 24
}

\begin{abstract}
Near-infrared spectra of planetary nebulae IC 418 and IC 5117 in the wavelength range $1.5-1.75 \mu \mathrm{m}$ observed with the United Kingdom Infrared Telescope (UKIRT) Cooled Grating Spectrometer (CGS2) are reported. More than 10 members of the Brackett line series are detected superposed on a strong continuum. Analysis of the spectra shows that the observed flux ratios of the Brackett lines are in good agreement with the recombination line theory after correction for extinction by the extinction law of Draine. A He I triplet line at $1.7 \mu \mathrm{m}$ is identified in both nebulae. A Mg I line at $1.504 \mu \mathrm{m}$, and a few Si I lines are also seen in the spectra. After subtraction of the nebular and central star continua by modeling the spectral energy distributions, a near-infrared excess is revealed. After correction for contribution from line emission, this excess can be explained by a hot dust component. However, the steep slope of the $H$-band spectrum of IC 418 remains unexplained and could be due to an unknown broad feature.
\end{abstract}

Subject headings: infrared: interstellar: continuum - planetary nebulae: individual (IC 418, IC 5117)

\section{INTRODUCTION}

Exploration of planetary nebulae in the infrared was started shortly after the advent of infrared technique. Gillett, Low, \& Stein (1967) detected strong $10 \mu \mathrm{m}$ emission from NGC 7027 and attributed this to dust thermal emission. The early infrared surveys confirmed that dust grains are present in many planetary nebulae (Cohen \& Barlow 1974). Over 1000 planetary nebulae have been detected by the IRAS survey in the farinfrared, and over 300 have photometric measurements in three or more of the four IRAS bands. It was found that the majority of planetary nebulae are strong infrared emitters and the infrared flux can be a significant fraction of the total energy output from a nebula.

Dust features commonly seen in circumstellar envelopes of asymptotic giant branch (AGB) stars are also detected in the infrared spectra of planetary nebulae, therefore suggesting an evolutionary link between the AGB and planetary nebula phases of evolution. The $9.7 \mu \mathrm{m}$ silicate feature commonly seen in oxygen-rich AGB stars was first identified in planetary nebulae by Aitken et al. (1979), and the $11.3 \mu \mathrm{m} \mathrm{SiC}$ feature commonly seen in carbon-rich AGB stars was detected by Willner et al. (1979) in IC 418 and IC 6572.

Because of the low temperature $(T<200 \mathrm{~K})$, the dust component revealed by the IRAS broad-band measurements cannot be responsible for the high level of flux observed in the near-infrared. From a sample of 80 planetary nebulae observed, Whitelock (1985) found that most nebulae show their $J H K$ colors dominated by hydrogen and helium plasma emission, and only 12 nebulae exhibit contributions from thermal emission of dust grains. Observations of compact (and presumably young) planetary nebulae suggest that the nearinfrared continuum is consistent with bound-free and free-free emissions from the gas component (Kwok, Hrivnak, \& Milone 1986).

Though near-infrared broad-band photometric observations have been useful in determining energy distributions of planetary nebulae, it is difficult to decipher the nature of the nearinfrared radiation on the basis of photometric data alone, as this near-infrared region covers various lines of atomic and molecular hydrogen, $\mathrm{He} \mathrm{I}$, and $\mathrm{He} \mathrm{II}$, as well as the forbidden lines. A good example is the spectrum of NGC 7027 from 0.9 to $2.7 \mu \mathrm{m}$, which was obtained with a Fourier Transform Spectrometer by Treffers et al. (1976). While the spectra in the mid-infrared for many planetary nebulae have been obtained from ground-based observations (e.g., 8-13 $\mu \mathrm{m}$, Aitken \& Roche 1982; Roche \& Aitken 1986) and the IRAS Low Resolution Spectrometer (LRS; Pottasch et al. 1986; Zhang \& Kwok 1990), fewer nebulae have been investigated using nearinfrared spectroscopy in the wavelength range of $1-5 \mu \mathrm{m}$. Current generations of cooled grating spectrometers have provided the possibility of obtaining spectra in the $1-5 \mu \mathrm{m}$ wavelength range with high sensitivity and resolution.

Broad-band near-infrared photometry of IC 418 has been obtained by Whitelock (1985), and that of IC 5117 has been obtained by Kwok et al. (1986) and van der Veen, Habing, \& Geballe (1989). These two objects have been extensively studied in the ultraviolet, optical, infrared, and radio. They both have carbon-rich chemical compositions in gas phase. While the carbon-rich grains of SiC (Willner et al. 1979; Pottasch 1987) and MgS (Forrest, Houck, \& McCarthy 1981; Moseley \& Silverberg 1985) are present in the far-infrared spectra of IC 418, the recent analysis of IRAS LRS of IC 5117 shows the coexistence of carbon-rich and oxygen-rich grains (Zhang \& Kwok 1990). Both of them exhibit far-infrared excesses. IC 418 shows an excess flux in $H$ and $K$ bands, which is attributed to a contribution from heated dust at a temperature of about $1350 \mathrm{~K}$ (Whitelock 1985). The H I Brackett and Pfund lines have been detected in the 2-4 $\mu \mathrm{m}$ spectrum of IC 418 by Willner et al. (1979). All these observations, when supplemented by near-infrared spectroscopy, will allow comprehensive pictures of the nebulae to be constructed.

In $\S 2$ we report near-infrared spectroscopic observations of IC 418 and IC 5117. The results are analyzed in $\$ 3$. Section 4 is devoted to discussions on overall spectral energy distributions of these two objects, from which the nature of continuum emission in the near-infrared is explored. A brief conclusion is given in $\S 5$.

\section{OBSERVATIONS AND RESULTS}

Near-infrared spectra of IC 5117 and IC 418 in the $H$ window were obtained on 1990 September 25 and 26 (UT), 
TABLE 1

LOG OF OBSERVATIONS

\begin{tabular}{|c|c|c|c|c|c|c|}
\hline \multirow[b]{2}{*}{ ОвJеCT } & \multirow[b]{2}{*}{$\begin{array}{l}\text { DATE } \\
\text { (UT) }\end{array}$} & \multirow[b]{2}{*}{$\begin{array}{l}\text { SPECTRAL } \\
\text { BAND }\end{array}$} & \multirow[b]{2}{*}{$\begin{array}{c}\text { OBSERVING } \\
\text { TIME } \\
\text { (minutes) }\end{array}$} & \multicolumn{3}{|c|}{ Calibration Star } \\
\hline & & & & Name & $\begin{array}{l}\text { Assumed } \\
\text { Magnitude }\end{array}$ & $\begin{array}{c}\text { Assumed } \\
\text { Temperature (K) }\end{array}$ \\
\hline IC $418 \ldots \ldots \ldots$ & 1990 Sep 26 & $H$ & 3 & BS 2918 & $H=4.68$ & 5900 \\
\hline IC $5117 \ldots \ldots \ldots$ & 1990 Sep 25 & $H$ & 4 & BS 8252 & $H=2.00$ & 4800 \\
\hline
\end{tabular}

respectively, at the $3.8 \mathrm{~m}$ United Kingdom Infrared Telescope (UKIRT) on Mauna Kea. The seven-channel cooled grating spectrometer (CGS2) at that facility in the $H(1.65 \mu \mathrm{m})$ wavelength band was used with a spectral resolution of $0.0039 \mu \mathrm{m}$. Spectra were sampled every one-third resolution elements, except at the ends of each spectrum. All spectra were obtained in a $5^{\prime \prime}$ aperture using chopper throws of $30^{\prime \prime} \mathrm{E}-\mathrm{W}$. Nearby bright stars BS 2918 and BS 8252 were observed as calibration sources for IC 418 and IC 5117, respectively. The spectra of IC 418 and IC 5117 were then calibrated by dividing by the spectra of the calibration stars and multiplying by blackbody functions assuming that the stellar continua are blackbodies at temperatures typical of their spectral types. Details are given in Table 1.

The spectra were reduced with the NOAO.ONEDSPEC package within the IRAF software. The theoretical wavelengths of the Brackett lines in the range $1.5-1.75 \mu \mathrm{m}$ were used to obtain a dispersion solution with a Chebyshev polynomial of the order of 2 and 3 for IC 418 and IC 5117, respectively. The spectra were then dispersion-corrected to a linear wavelength coordinate system. The resultant spectra of IC 418 and IC 5117 are shown in Figures 1 and 2, respectively, where the flux is plotted against wavelength.

The hydrogen Brackett recombination lines, corresponding to transitions from energy levels 10 up to 21 to level 4 , are prominent in the spectra of the nebulae. A triplet line of $\mathrm{He} \mathrm{I}$ $3 p^{3} P-4 d^{3} D$ at $1.7002 \mu \mathrm{m}$ is identified in the spectra of the two nebulae. A line of $\mathrm{Mg} \mathrm{I} 4 s^{3} S-4 p^{3} P$ at $1.504 \mu \mathrm{m}$ is identified in the spectra of IC 418 and IC 5117. Some weak features are also seen in both the spectra (see below).

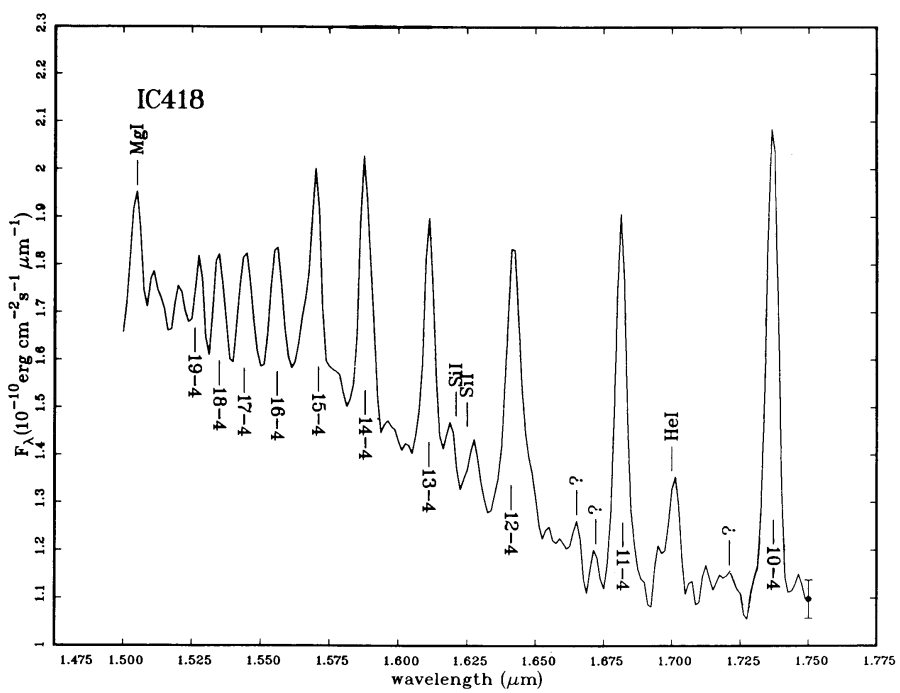

FIG. 1.-The CGS2 spectrum of IC 418 in the $H$ band. A typical error bar is indicated at the long-wavelength end of the spectrum.
The task SPLOT in the ONEDSPEC package of the IRAF was used to measure the fluxes in lines. Two methods were applied to obtain the line fluxes: (1) summing up the pixel intensities above the continuum within two marked wavelengths and (2) using the deblend routine which performs a nonlinear least-squares Gaussian fit to the line(s). The results from the two methods were then weight-averaged to give a final measurement. The measured line fluxes are presented in Table 2, where all the line fluxes are normalized to the Brackett $10-4$ line, B10, and a $1 \sigma$ error is also assigned to each measured line.

\section{LINE EMISSION}

Three separate components contribute to the observed emission spectrum of planetary nebulae. Photospheric continuum emission from the central star plus nebular two-photon, bound-free, and free-free emission dominates the continuum radiation seen in the ultraviolet. In the visible, nebular emission in the form of recombination and collisionally excited lines and hydrogen bound-free continuum emission are the most important. In the far-infrared, most of observed fluxes originate from dust thermal continuum emission. In the nearinfrared, both hot dust and nebular emission can be important. Previous broad-band photometry in the near-infrared for these two objects cannot give a definitive answer to the nature of their near-infrared emission. The near-infrared spectra reported here will allow us to study quantitatively both line and continuum emission in the wavelength range $1.5-1.75 \mu \mathrm{m}$. In this section we will first discuss emission lines.

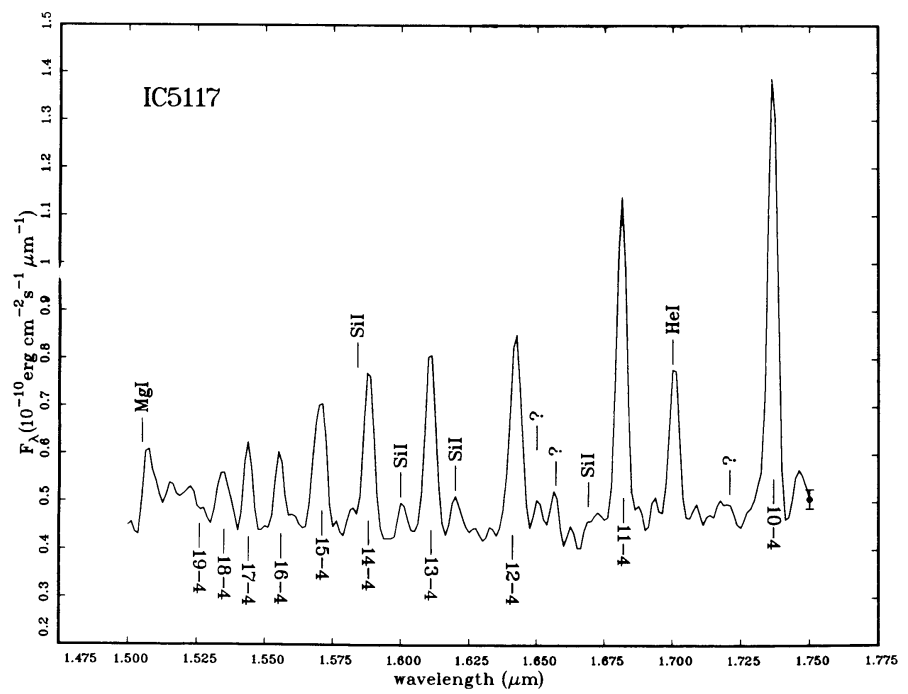

FIG. 2.-The CGS2 spectrum of IC 5117 in the $H$ band. A typical error bar is indicated at the long-wavelength end of the spectrum. 
TABLE 2

LINE FLUXES

\begin{tabular}{|c|c|c|c|c|c|c|}
\hline \multirow[b]{2}{*}{ SPECIES } & \multicolumn{3}{|c|}{ IC 418} & \multicolumn{3}{|c|}{ IC 5117} \\
\hline & Observed & Corrected & Predicted & Observed & Corrected & Predicted \\
\hline \multicolumn{7}{|l|}{ H I Brackett lines: } \\
\hline B10 ............. & 1.00 & 1.00 & 1.00 & 1.00 & 1.00 & 1.00 \\
\hline B11 ............ & $0.70 \pm 0.070$ & 0.70 & 0.75 & $0.72 \pm 0.070$ & 0.74 & 0.74 \\
\hline $\mathrm{B} 12 \ldots \ldots \ldots \ldots$ & $0.61 \pm 0.090$ & 0.62 & 0.57 & $0.45 \pm 0.070$ & 0.46 & 0.57 \\
\hline $\mathrm{B} 13 \ldots \ldots \ldots \ldots \ldots$ & $0.36 \pm 0.060$ & 0.37 & 0.45 & $0.37 \pm 0.060$ & 0.39 & 0.45 \\
\hline B14 …................. & $0.48 \pm 0.080$ & 0.48 & 0.36 & $0.34 \pm 0.060$ & 0.36 & 0.36 \\
\hline $\mathrm{B} 15 \ldots \ldots \ldots \ldots$ & $0.27 \pm 0.060$ & 0.27 & 0.29 & $0.34 \pm 0.070$ & 0.37 & 0.30 \\
\hline B16 …................. & $0.24 \pm 0.060$ & 0.24 & 0.24 & $0.14 \pm 0.034$ & 0.15 & 0.25 \\
\hline $\mathrm{B} 17 \quad \ldots \ldots \ldots \ldots \ldots \ldots \ldots$ & $0.24 \pm 0.060$ & 0.24 & 0.20 & $0.14 \pm 0.040$ & 0.15 & 0.21 \\
\hline B18 …................ & $0.15 \pm 0.040$ & 0.16 & 0.17 & $0.11 \pm 0.030$ & 0.12 & 0.17 \\
\hline 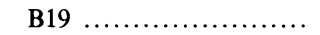 & $0.08 \pm 0.020$ & 0.10 & 0.15 & $\ldots$ & $\ldots$ & 0.15 \\
\hline B20 ....... & $0.04 \pm 0.010$ & 0.04 & 0.13 & $\ldots$ & $\ldots$ & 0.13 \\
\hline B21 ................ & $0.01 \pm 0.003$ & 0.01 & $\ldots$ & $\ldots$ & $\ldots$ & $\ldots$ \\
\hline \multicolumn{7}{|l|}{ He I: } \\
\hline $1.7002 \mu \mathrm{m}$ & $0.29 \pm 0.07$ & 0.29 & $\ldots$ & $0.32 \pm 0.040$ & 0.32 & $\ldots$ \\
\hline \multicolumn{7}{|l|}{ Mg I: } \\
\hline $1.5047 \mu \mathrm{m} \ldots \ldots$. & $0.16 \pm 0.03$ & 0.16 & $\ldots$ & $0.10 \pm 0.025$ & 0.11 & $\ldots$ \\
\hline
\end{tabular}

Notes.-Fluxes are normalized to the $\mathrm{H}$ I B10 line flux. The observed $\mathrm{H}$ I B10 line fluxes are $(5.04+0.50) \times 10^{-13}$ and $(4.02 \pm 0.40) \times 10^{-13} \mathrm{ergs} \mathrm{cm}^{-2} \mathrm{~s}^{-1}$ for IC 418 and IC 5117 , respectively. The extinction-corrected $\mathrm{H} \mathrm{I} \mathrm{B10} \mathrm{line} \mathrm{fluxes} \mathrm{are}$ $(5.55 \pm 0.60) \times 10^{-13}$ and $(6.10 \pm 0.60) \times 10^{-13} \mathrm{ergs} \mathrm{cm}^{-2} \mathrm{~s}^{-1}$ for IC 418 and IC 5117 , respectively.

\subsection{The Hydrogen Lines}

An outstanding feature of the CGS2 spectra of IC 418 and IC 5117 is the series of Brackett emission lines superposed on a continuum. In order to correct for the differential extinction within the series, we have adopted the near-infrared extinction law of Draine (1990):

$$
A_{\lambda}=1.3 E(B-V)(\lambda / \mu \mathrm{m})^{-1.75},
$$

where $E(B-V)$ is the color excess. Three methods were used to derive the color excess, $E(B-V)$, for the objects: (1) by comparison of the radio continuum flux density with the $\mathrm{H} \beta$ flux, (2) by the Balmer decrements, and (3) by the so-called $2200 \AA$ dip seen in the IUE spectra. Using the average of the three methods, we derived $E(B-V)$ values of 0.21 and 0.88 for IC 418 and IC 5117, respectively. The observed Brackett line fluxes were then corrected for extinction using these values of $E(B-V)$ and equation (1). The corrected fluxes of the measured lines are listed in Table 2.

The $\mathrm{H} \mathrm{I}$ emission lines in a planetary nebula arise from the recombination of protons and electrons. Extensive calculations have been carried out on the intensity ratios of recombination lines under various values of electron densities and temperatures. To test the recombination line theory, we have compared the corrected ratios of the series of the Brackett lines over B10 with the predictions from the recombination line theory. We have adopted the electron densities $n_{e}=1.02 \times 10^{4}$ and $3.4 \times 10^{4} \mathrm{~cm}^{-3}$ and the electron temperatures $T_{e}=8300$ and 11,800 K for IC 418 (Shaw \& Kaler 1989) and IC 5117 (Aller \& Czyzak 1983), respectively (see Table 3). The predicted flux ratios of the series Brackett lines over B10 can then be obtained from the recombination line theory (case B) developed by Brocklehurst (1971) and Giles (1977). The line ratios of $I(n-4) / I(n-2)$ are given for $n=5(1) 10,15,20, T_{e}=5 \times 10^{3}$, $10^{4}, 2 \times 10^{4} \mathrm{~K}, n_{e}=0,10^{2}, 10^{4}, 10^{6} \mathrm{~cm}^{-3}$, and for the limit $n_{e} \rightarrow \infty$ by Giles (1977), while the line ratios of $I(n-2) / I(4-2)$ for $3 \leq n \leq 40, T_{e}=5 \times 10^{3}, 10^{4}, 2 \times 10^{4} \mathrm{~K}, n_{e}=10^{2}, 10^{3}$, $10^{5}, 10^{6} \mathrm{~cm}^{-3}$ are tabulated by Brocklehurst (1971). Linear interpolations have been applied to obtain the line ratios of the
Brackett series in the observed spectra from $n=10$ to $n=20$ for the adopted $n_{e}$ and $T_{e}$. These theoretically predicted line ratios are also listed in Table 2. In Figure 3, we have plotted the predicted ratios together with the observed ratios corrected for extinction as a function of the upper level quantum numbers. From Figure 3, it is seen that the predicted ratios are in agreement with the corrected ratios for IC 418 and IC 5117 within the observational uncertainties.

From the observed IC $5117 \mathrm{H} \beta$ flux of $\log \mathrm{H} \beta=-11.4$ (cf. Pottasch 1984) we derive an extinction-corrected $\mathrm{H} \beta$ flux of $7.67 \times 10^{-9} \mathrm{ergs} \mathrm{cm}^{-2} \mathrm{~s}^{-1}$, using $E(B-V)=0.88$. This results in a $\mathrm{B} 10$ to $\mathrm{H} \beta$ flux ratio of 0.008 . This is in good agreement with the theoretical ratio of 0.0088 derived from the recombination line theory for the adopted electron density and temperature (Brocklehurst 1971; Giles 1977).

The $\mathrm{H} \beta$ flux of IC 418 corrected for extinction $E(B-V)=0.21$ is $5.33 \times 10^{-10}$ ergs $\mathrm{cm}^{-2} \mathrm{~s}^{-1}$, taking the observed $\mathrm{H} \beta$ flux of $\log \mathrm{H} \beta=-9.58$ (cf. Pottasch 1984). The

TABLE 3

Physical Parameters

\begin{tabular}{|c|c|c|}
\hline Parameter & IC 418 & IC 5117 \\
\hline$n_{e}\left(10^{3} \mathrm{~cm}^{-3}\right)$. & 10.2 & 34.0 \\
\hline$T_{e}\left(10^{4} \mathrm{~K}\right) \ldots \ldots \ldots \ldots \ldots \ldots \ldots \ldots \ldots \ldots \ldots \ldots \ldots$ & 0.83 & 1.18 \\
\hline$y_{1}+\cdots+\cdots$ & 0.076 & 0.102 \\
\hline (. & 0.000 & 0.008 \\
\hline$E(B-V)$ & 0.21 & 0.88 \\
\hline$\theta_{*}\left(10^{-7} \operatorname{arcsec}\right) \ldots \ldots$ & 56.0 & 9.73 \\
\hline$T_{*}^{*}\left(10^{3} \mathrm{~K}\right) \ldots \ldots \ldots \ldots$ & 40.2 & 56.7 \\
\hline$T_{d}^{*}(\mathrm{~K}) \ldots \ldots$ & 177 & 189 \\
\hline$\tau\left(10^{-4}\right)$ & 4.26 & 63.8 \\
\hline$F_{\mathrm{H} \beta}\left(10^{-10} \mathrm{ergs} \mathrm{cm}^{-2} \mathrm{~s}^{-1}\right)$ & 5.33 & 0.77 \\
\hline$F_{\mathrm{BB}} / F_{\mathrm{H} \beta} \ldots \ldots \ldots \ldots \ldots \ldots \ldots \ldots \ldots \ldots \ldots \ldots \ldots$ & 205 & 170 \\
\hline$F_{t} / F_{\mathrm{H} \beta}+\cdots \cdots \cdots \cdots \cdots$ & 187 & 193 \\
\hline$F_{*} / F_{\mathrm{H} \beta}$ & 106 & 50 \\
\hline 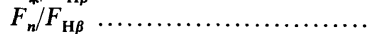 & 14 & 14 \\
\hline$F_{1} / F_{\mathrm{H} \beta} \ldots \ldots \ldots \ldots \ldots \ldots \ldots \ldots \ldots \ldots \ldots \ldots \ldots \ldots$ & 14 & 37 \\
\hline$F_{\mathrm{IR}} / F_{\mathrm{H} \beta} \ldots \ldots \ldots \ldots \ldots \ldots \ldots \ldots \ldots$ & 54 & 93 \\
\hline 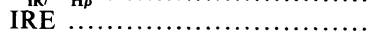 & 1.9 & 2.2 \\
\hline
\end{tabular}




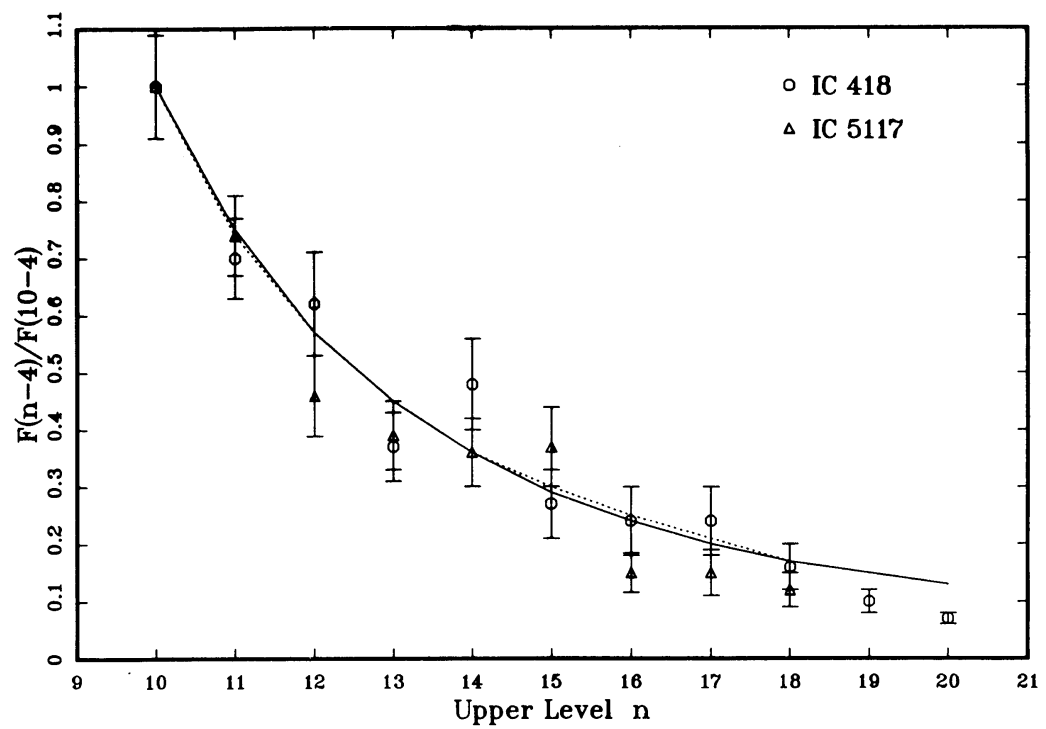

Fig. 3.-The Brackett line ratios corrected for the extinction as a function of the upper level quantum number for IC 418 and IC 5117, respectively. The corresponding theoretical predicted line ratios (case B) are also plotted for comparison.

observed extinction-corrected B10 line flux in our spectrum for IC 418 is thus about 0.001 of the $\mathrm{H} \beta$ flux. However, the recombination line theory predicts this ratio at 0.0095 for the electron density and temperature of IC 418. This discrepancy is due to the fact that the used entrance aperture of $5^{\prime \prime}$ is smaller than the diameter of $12^{\prime \prime}$ of IC 418 , much of the emission was missed. The CCD image of IC 418 through $\mathrm{H} \alpha$ line shows a limb-brightened ring structure (Balick 1987). It is likely that the $\mathrm{H}$ I $\mathrm{H} \beta$ and $\mathrm{B} 10$ lines have the same spatial distribution as the $\mathrm{H} \alpha$ line. Our near-infrared spectrum of IC 418 is likely to have missed the brightest outer ring part of the emission.

\subsection{Helium Lines}

A feature at $1.7 \mu \mathrm{m}$ is clearly seen in the CGS2 spectra of the two objects. This is identified as a $\mathrm{He} \mathrm{I}$ triplet line, corresponding to the transition $3 p^{3} P-4 d^{3} D$ at $1.7002 \mu \mathrm{m}$.

Theory (Robinns 1968) predicts the He I line at $1.7 \mu \mathrm{m}$ at a dereddened flux of 0.072 in units of $\mathrm{He}$ I $\lambda 4471$ flux. The observed ratio of $I_{\lambda 1.7} / I_{\lambda 4471}$ can be derived using the following formula:

$$
\frac{I_{\lambda 1.7}}{I_{\lambda 4471}}=\frac{I_{\lambda 1.7}}{B 10} \frac{B 10}{\mathrm{H} \beta} \frac{\mathrm{H} \beta}{I_{\lambda 4471}} .
$$

Taking the observed unextinguished ratio $I_{\lambda 1.7} / \mathrm{B} 10=0.29$ and 0.32 (see Table 2), the expected ratio $\mathrm{B} 10 / \mathrm{H} \beta=0.0095$ and 0.0088 (see $\S 3.1$ above), and the observed unextinguished ratio $I_{\lambda 4471} / \mathrm{H} \beta=0.035$ and 0.044 (Aller \& Czyzak 1979, 1983), we obtain the ratio $I_{\lambda 1.7} / I_{\lambda 4471}=0.079$ and 0.064 for IC 418 and IC 5117, respectively. The observational results show a general agreement with the theory within the uncertainties. This ratio of He I $1.7 \mu \mathrm{m}$ to $\lambda 7741$ flux for NGC 7027 was observed at 0.056 by Treffers et al. (1976).

\subsection{Other Emission Lines}

A feature at $1.504 \mu \mathrm{m}$ seen in both the spectra of IC 418 and IC 5117 is believed to be the $\mathrm{Mg} \mathrm{I} 4 s^{3} S-4 p^{3} P$ line. Though the $\mathrm{Mg}$ II $2800 \AA$ line was detected in the high-resolution International Ultraviolet Explorer Satellite (IUE) spectrum of IC 418 (Middlemass 1989), this neutral atomic Mg I line is for the first time detected in planetary nebulae. This line was seen in the spectrum of IRC + 10420 (an F8 supergiant) together with a Mg I $1.574 \mu \mathrm{m}$ line (Thompson \& Boroson 1977). As suggested by Thompson and Boroson, the $\mathrm{Mg}$ I lines are produced by UV fluorescence. Absorption of a UV photon at $3096 \AA$ from the metastable triplet state $3 p{ }^{3} P^{o}$ will populate the upper $4 d$ ${ }^{3} \mathrm{D}$ state and follow a cascade to the lower $4 p{ }^{3} P^{o}$ and $4 s{ }^{3} S$ states. The $\mathrm{Mg}$ I $1.574 \mu \mathrm{m}$ line should be emitted due to the $4 d$ ${ }^{3} D-4 p{ }^{3} P^{o}$ transition, which may populate the $4 p{ }^{3} P^{o}$ state. It is possible that the $1.574 \mu \mathrm{m}$ line is blended with the B15 line. The question whether the $1.574 \mu \mathrm{m}$ line exists and how strong it would be can only be answered until high-resolution spectra are available. The $4{ }^{3} P^{o}$ state can also be populated via the absorption of a $3336 \AA$ photon from the metastable state $3 p{ }^{3} P^{o}$ to the $5 s{ }^{3} S$ state followed by the $5 s{ }^{3} S-4 p{ }^{3} P^{o}$ transition, which emits the $2.487 \mu \mathrm{m}$ line outside the wavelength range of the $H$-band spectrum.

A series of features at $1.583,1.596,1.621$, and $1.668 \mu \mathrm{m}$ in the spectrum of IC 5117 can be identified with the Si I lines on the basis of agreement in wavelength separations and their wavelength positions.

Inspection of the Grotrian diagrams of Si I (Bashkin \& Stoner 1975) suggests that the Si I lines are probably excited by UV fluorescent mechanisms. The ground state is a triplet $3 p^{2}{ }^{3} P$ state, while a singlet $3 p^{2}{ }^{1} D$ state is metastable. The absorption of a photon at about $1846 \AA$ will excite $\mathrm{Si}$ I from the ground state to either the $5 s{ }^{3} P^{o}\left(54362 \mathrm{~cm}^{-1}\right)$ or the $3 d^{3} D^{o}$ $\left(54216 \mathrm{~cm}^{-1}\right)$ state. Two sets of the triplet lines are produced following the transitions $5 s^{3} P^{o}-4 p{ }^{3} D(1.596 \mu \mathrm{m})$ and $3 d^{3} D^{o}-4 p{ }^{3} D(1.621$ and $1.668 \mu \mathrm{m})$. In the former triplet, the weaker component at $1.556 \mu \mathrm{m}(J=2-2)$ is probably blended with the $\mathrm{H}$ I B16 line, while in the latter, the component at $1.688 \mu \mathrm{m}(J=3-3)$ appears to be stronger than the one at $1.621 \mu \mathrm{m}(J=1-1)$. The singlet $4 d^{1} D-4 p^{1} D$ line at $1.583 \mu \mathrm{m}$ is also due to UV fluorescence. The absorption of a $1992 \AA$ photon may populate the $4 d^{1} D$ state. The $1.583 \mu \mathrm{m}$ line is then produced via the transition $4 d^{1} D-4 p^{1} D$.

The two features at 1.621 and $1.625 \mu \mathrm{m}$ in the spectrum of IC 418 can be tentatively identified with the $\mathrm{Si}$ I transitions of $3 d^{3} D^{o}-4 p{ }^{3} D$. This suggested identification is less certain than 
that in the case of IC 5117 since fewer lines are seen and the strongest component in this triplet at $1.668 \mu \mathrm{m}$ is barely recognizable.

Because the ionization potentials of $\mathrm{Mg}$ I and $\mathrm{Si}$ I are 7.64 and $8.1 \mathrm{eV}$, they are not expected to be present in the ionized $\mathrm{H}$ II region of the nebulae. The $\mathrm{Mg}$ I and $\mathrm{Si}$ I lines could originate from a neutral region around the ionized nebulae or some dense condensations within the nebulae.

Before the above features were identified, a careful exercise was undertaken to examine the effects of absorption features appearing in the spectra of the reference stars. First of all, we noticed that the $\mathrm{Mg}$ I and $\mathrm{Si}$ I lines are clearly seen in emission in the raw spectra of the two planetary nebulae. An absorption feature of $\mathrm{Mg}$ I $1.504 \mu \mathrm{m}$ line is probably present in the spectra of the reference stars of spectral type G. We replaced these absorption features by an interpolation of the continuum in the reference spectra before the ratioing of the raw spectra of the program stars by the reference spectra was carried out. We are, therefore, convinced that the $\mathrm{Mg}$ I and $\mathrm{Si}$ I lines seen in the two nebulae are real and are not the result of the ratioing procedure.

\subsection{The Unidentified Lines}

Three unidentified lines at $1.650,1.655$, and $1.721 \mu \mathrm{m}$ may be present in the spectrum of IC 5117, while three unidentified lines at $1.665,1.672$, and $1.721 \mu \mathrm{m}$ may be present in that of IC 418. Further investigations with higher sensitivity and resolution are needed.

\subsection{A Comment on the PAH 1.69 Micron Feature}

Several unidentified infrared features (UIF) at 3.3, 5.6, 6.9, 7.7, 8.7, and $11.3 \mu \mathrm{m}$ are detected for IC 418 and IC 5117 (Cohen et al. 1986 and the references therein). The $3.3 \mu \mathrm{m}$ feature in IC 418 is also detected by Martin (1987). The 7.7 and $11.3 \mu \mathrm{m}$ features are seen in the IRAS LRS spectrum of IC 5117 (Zhang \& Kwok 1990). Large polycyclic aromatic hydrocarbon (PAH) molecules are believed to be the carriers of these UIF features (Leger \& Puget 1984; Allamandola, Tielens, \& Barker 1985). The PAH hypothesis predicts that a band at 1.69 $\mu \mathrm{m}$, which is a second overtone of the fundamental $\mathrm{CH}$ mode, is expected to be observed at a band strength 0.03 times as strong as the $3.3 \mu \mathrm{m}$ feature for a color temperature of $700 \mathrm{~K}$ (Leger, d'Hendecourt, \& Defourneau 1989). Taking the $3.3 \mu \mathrm{m}$ band strength of $1.8 \times 10^{-11}$ (Martin 1987) and $8.5 \times 10^{-12}$ (Cohen et al. 1986) ergs cm $\mathrm{cm}^{-2} \mathrm{~s}^{-1}$, we estimate that the $1.69 \mu \mathrm{m}$ band would have a strength of $5.1 \times 10^{-13}$ and $2.6 \times 10^{-13}$ ergs $\mathrm{cm}^{-2} \mathrm{~s}^{-1}$ for IC 418 and IC 5117 , respectively. There is apparently no such a feature detected in the $H$ window CGS2 spectra of the two nebulae at the noise levels of $(2-3) \times 10^{-12}$ ergs $\mathrm{cm}^{-2} \mathrm{~s}^{-1} \mu \mathrm{m}^{-1}$. Careful inspection of the spectrum of NGC 7027 from 0.9 to $2.7 \mu$ m (Treffers et al. 1976) shows no indication of this feature at $1.69 \mu \mathrm{m}$ either. Longer integration time is needed to obtain a spectrum with a higher $\mathrm{S} / \mathrm{N}$ ratio in order to test the existence of the $1.69 \mu \mathrm{m}$ feature predicted by the PAH hypothesis.

\section{CONTINUUM EMISSION}

In addition to the emission lines discussed in the last section, strong continuum emission is detected in both IC 418 and IC 5117. In the near-infrared region, several emission processes contribute to the continuum emission. These include boundfree and free-free emissions from hydrogen and helium, as well as thermal emission from dust. In order to determine the rela- tive contributions of these processes, we have constructed overall spectral energy distributions (SED) of the two nebulae from 0.1 to $100 \mu \mathrm{m}$.

\subsection{IC 418}

\subsubsection{Energy Distribution}

For IC 418, we compiled observational data from various sources. The merged low-resolution IUE images SWP 6651 and LWR 2257 of IC 418 were extracted and processed at the IUE Regional Data Analysis Facility ${ }^{1}$ (IUE-RDAF) located at the Center for Astrophysics and Space Astronomy (CASA), The University of Colorado. The images were absolutely calibrated and corrected for camera sensitivity degradation (LWR only) and then joined at $1975 \AA$. The optical continuum fluxes measured with narrow-band photometry were taken from Kohoutek \& Martin (1981). The optical emission-line fluxes were from Aller \& Czyzak $(1979,1983)$. The near-infrared $J H K$ data were measured by Whitelock (1985), and the $L$-band data were taken from Cohen \& Barlow (1974). The IRAS Point Source Catalog (PSC Version 2, 1988) fluxes were corrected for color using the procedure described in Kwok et al. (1986). The LRS spectrum of IC 418 was taken from the tape version of the LRS Catalog (Atlas of Low Resolution IRAS Spectra 1986). The baseline of the LRS spectrum was removed and the spectrum was recalibrated according to Volk \& Cohen's scheme (1989).

To incorporate the CGS2 spectrum of IC 418 into the SED, one has to correct for the fact that the entrance aperture of the CGS2 spectrometer used was $5^{\prime \prime}$, less than the diameter of $12^{\prime \prime}$ of this nebula. A correction factor of 5 has been applied to scale the CGS2 spectral flux level to the entire nebula in order to match the $H$-band photometric flux measured by Whitelock.

After correcting for the extinction, we plotted the energy distribution ( $\lambda F_{\lambda}$ vs. $\lambda$ ) of IC 418 from 0.1 to $100 \mu \mathrm{m}$ in Figure 4. A model consisting of three components, namely a central star, a gaseous nebula, and a dust shell, as described in Zhang \& Kwok (1991), is fitted to the observed SED. In this model, the nebular bound-free and free-free emission from $\mathrm{H} \mathrm{I}, \mathrm{He} \mathrm{I}$, and $\mathrm{He} \mathrm{II}$, and the hydrogen two-photon emission have been taken into account in the effective gaseous continuum emission coefficient $\left(\gamma_{\text {eff }}\right)$. Calculations of $\gamma_{\text {eff }}$ as a function of the electron density, $n_{e}$, the electron temperature $T_{e}$, the helium abundance, $y_{1}=\mathrm{He}^{+} / \mathrm{H}^{+}$, and $y_{2}=\mathrm{He}^{++} / \mathrm{H}^{+}$, are based on the formalism of Brown \& Mathews (1970), with our own extension to $1000 \AA$ on the short-wavelength side and to $20 \mu \mathrm{m}$ on the long-wavelength side. The model curves corresponding to the three components and the synthesized model SED are also shown in Figure 4.

From the model fitting, we obtain the angular radius $\left(\theta_{*}\right)$ and the temperature $\left(T_{*}\right)$ of the central star, along with the dust temperature $\left(T_{d}\right)$, the optical depth at $60 \mu \mathrm{m}(\tau)$ of the dust shell, the ratios of the nonionizing flux from the central star $\left(F_{*}\right)$, the flux of the nebular bound-free, free-free, and two-photon continuum $\left(F_{n}\right)$, the flux of all the observed emission lines $\left(F_{l}\right)$, the flux due to dust thermal emission prominent in the IRAS bands $\left(F_{\mathrm{IR}}\right)$, the total measured flux $\left(F_{t}\right)$, which is a summation of the above flux components, and the total flux of the central star for a blackbody $\left(F_{\mathrm{BB}}\right)$, over the $\mathrm{H} \beta$ flux. The far-infrared excess (IRE), defined as a ratio of total infrared flux seen by the $I R A S$ over the Ly $\alpha$ flux predicted from the optically thin radio

\footnotetext{
${ }^{1}$ The Colorado IUE-RDAF is supported by NASA contract number NAS 5-28731.
} 


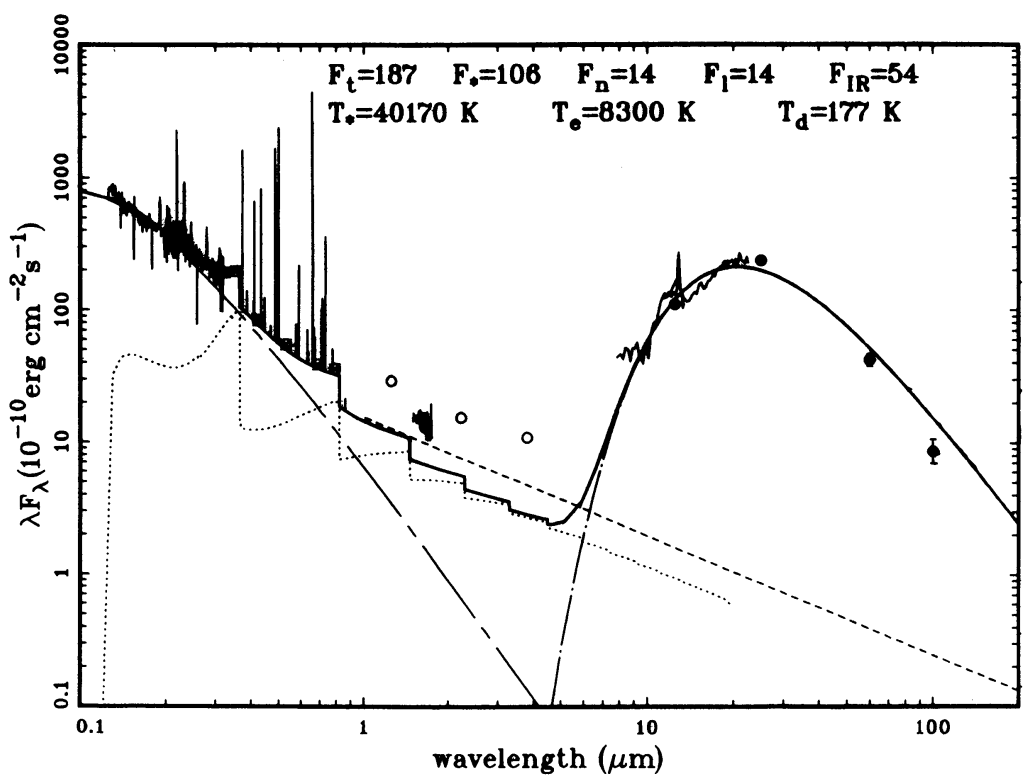

FIG. 4. - The observed spectral energy distribution of IC 418 corrected for the extinction in the wavelength range $0.1-100 \mu \mathrm{m}$. Thin lines around 0.1 and $10 \mu \mathrm{m}$ are the IUE and IRAS LRS spectra, respectively. Dots are color-corrected IRAS survey measurements. Open circles are near-infrared $J H K L$ broad-band measurements. Crossed squares indicate optical continuum measurements. Vertical lines illustrate various emission lines in the optical. Model curves for the stellar, nebular, and dust components are shown as chain-dashed, dotted, and chain-dotted lines, respectively. Thick solid lines show the synthesized spectral energy distribution. Dashed lines indicate the level of free-free emission extrapolated from the optically thin radio continuum flux density. The best-fit parameters, $T_{*}$ and $T_{d}$, are shown along with $T_{e}$ at the top part of the diagram. The integrated total observed flux $\left(F_{t}\right)$, the nonionizing flux of the central star $\left(F_{*}\right)$, the nebular gaseous continuum flux $\left(F_{n}\right)$, the flux in the observed emission lines $\left(F_{l}\right)$, and the flux in far-infrared $\left(F_{\mathrm{IR}}\right)$ are given above the row of the temperatures in units of $F_{\mathrm{H} \beta}$. The CGS2 spectrum in the $H$ band is incorporated in this diagram. The flux level is corrected for the effect of the aperture smaller than the nebular size (see the text).

continuum flux density, can also be derived at 1.9 for IC 418 . The above derived physical parameters are summarized in Table 3, together with $n_{e}, T_{e}$, the $\mathrm{He}^{+}$abundance $y_{1}$, and the $\mathrm{He}^{++}$abundance $y_{2}$ (adopted from Shaw \& Kaler 1989), and the color excess, $E(B-V)$.

The central star temperature of IC 418 was derived at 36,000 $\mathrm{K}$ by Mendez et al. (1988) using model atmospheres to fit the observed stellar photospheric absorption-line profiles. A temperature of $36,000 \mathrm{~K}$ is derived by Preite-Martinez et al. $(1989)$ using the energy-balance method, and a temperature of 38,000 $\mathrm{K}$ is obtained by Shaw \& Kaler (1989) using the Zanstra method. The central star temperature of $40,000 \mathrm{~K}$ derived from our best fitting to the observed SED is about $5 \%-10 \%$ higher than those derived by other methods. It is very close to the Zanstra temperature by Shaw and Kaler, who derived a more accurate central star magnitude. The photometric variations of the central star (Mendez 1989; Mendez et al. 1988) may also cause the discrepancy.

An estimate of the free-free continuum extrapolated from the radio continuum measurements at $6 \mathrm{~cm}$ is also plotted (dashed line) in Figure 4. We note that this extrapolation is in rough agreement with the nebular model continuum. A mismatch between the modeled and the extrapolated nebular continuum in the infrared is about a factor of less than 2. Considering the extrapolation is done over three orders of magnitude in wavelength, this is not unexpected. In the extrapolation, we use a power-law index of -0.1 for the optically thin radio continuum spectrum. This index originates from the power-law fitting of the Gaunt factor in the free-free absorption coefficient for the radio wavelengths (Mezger and Henderson 1967). The discrepancy is primarily caused by the fact that the radio power-law index of -0.1 is no longer valid for the wavelength range in the infrared.

\subsubsection{Origin of the Near-Infrared Excess}

The broad-band near-infrared photometry suggests that there exists near-infrared excess emission over the contributions from the central star, the nebular plasma and the cool dust (177 K indicated by the IRAS measurements) for IC 418 . An excess emission from 2 to $4 \mu \mathrm{m}$ was first noticed by Higgs (1971 and references therein). The excess emission from IC 418 was discussed by Willner et al. (1979) in terms of thermal radiation due to hot dust, free-free radiation produced by interactions between free electrons and ions $\mathrm{H}^{-}$and between free electrons and $\mathrm{H}_{2}^{-}$, and stellar radiation scattered by dust particles. They found that this excess radiation cannot be satisfactorily explained with any of the above three mechanisms. This excess was also noticed by Whitelock (1985). She assigned the excess emission in the $\mathrm{H}$ and $\mathrm{K}$ bands to hot dust with a temperature of $1350 \mathrm{~K}$. Phillips et al. (1984) did a scanning of IC 418 in the $J, H, K$, and $L$ bands. They showed that the $\mathrm{H}$-band excess emission is coincident with the ionized zone and suggested that it represents the Rayleigh-Jeans tail of the central star. They argued that while the $L$-band excess emission originates from cooler dust located inside the ionized zone, the $K$-band excess emission arises from very small, hotter dust located outside the ionized zone.

To investigate the nature of the excess emission, we have subtracted the model continuum in the near-infrared from the $J-, H-, K-$, and $L$-band measurements. The near-infrared excesses are then found to be $16.5,4.73,4.69$, and $2.14 \times 10^{-10}$ ergs $\mathrm{cm}^{-2} \mathrm{~s}^{-1}$ at the $J, H, K$, and $L$ bands, respectively. They are plotted as a function of wavelength in Figure 5, where the corresponding excess seen in the CGS2 spectrum was obtained by scaling the spectrum by a factor of 5 (see above). The most striking characteristics of this excess emission is a steep slope seen in the $H$ band. 


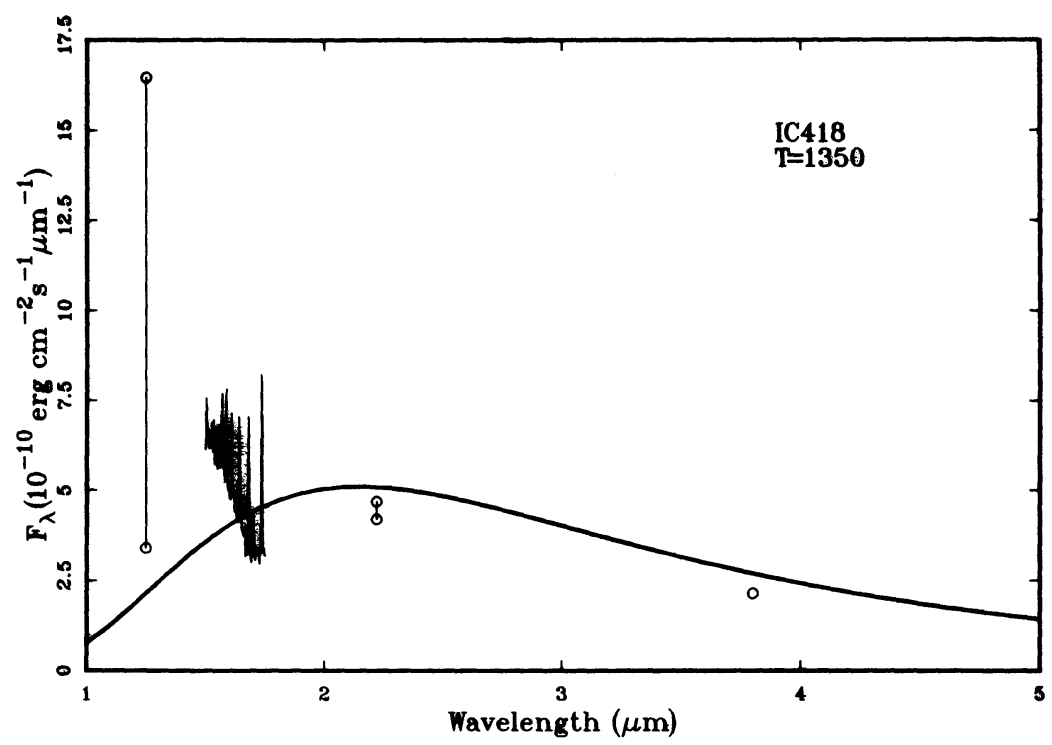

Fig. 5.-The near-infrared excess emission of IC 418 plotted against wavelengths. The two circles joined by a vertical line are band excesses before and after correction for line emission. For $H$ band, the upper excess point is from broad-band photometry and the lower point from the spectrum value at $1.65 \mu \mathrm{m}$. The CGS2 spectrum is also shown after subtraction of the model nebular continuum. The solid line is a $1350 \mathrm{~K}$ blackbody curve.

Contributions from line emission to the broad-band nearinfrared photometric measurements must account for at least part of the excess emission. The most important lines are (1) $\mathrm{H}$ I $\mathrm{Pa} \beta(1.282 \mu \mathrm{m}), \mathrm{H}$ I Pa $\gamma(1.094 \mu \mathrm{m}), \mathrm{He}$ II $7-5(1.165 \mu \mathrm{m})$, and He I $2{ }^{3} S-2{ }^{3} P(1.083 \mu \mathrm{m})$ in $J$ band; (2) the lines seen in the CGS2 spectra in the $H$ band; (3) $\mathrm{H}$ I Bry $(2.166 \mu \mathrm{m}), \mathrm{He}$ I $2{ }^{1} S-$ $2{ }^{1} P(2.058 \mu \mathrm{m})$ in $K$ band.

The $J$-band excess emission is believed to be dominated by the $\mathrm{He}$ I $1.083 \mu \mathrm{m}$ line emission (Whitelock 1985). This line is, however, at the edge of the $J$-band filter with about $58 \%$ of its light being transmitted. Careful assessment of this line contribution is therefore necessary. Scrimger (1984) observed line ratios of $[I(\mathrm{~Pa} \beta) / I(\mathrm{H} \beta)]_{\mathrm{obs}}=0.34$ and $[I(1.083) / I(\mathrm{H} \beta)]_{\mathrm{obs}}=$ 1.48 in IC 418 . Using the $E(B-V)$ value above, these ratios corrected for extinction are $[I(\mathrm{~Pa} \beta) / I(\mathrm{H} \beta)]_{\text {true }}=0.20$ and $[I(1.083) / I(\mathrm{H} \beta)]_{\text {true }}=0.90$. For the Pay line we use the recombination line theory to obtain $[I(\mathrm{~Pa} \gamma) / I(\mathrm{H} \beta)]_{\text {true }}=0.09$. Since there is no He II line present in IC 418 (Aller \& Czyzak 1979), He II 7-5 line does not contribute to the $J$-band flux. As the He I $1.7 \mu \mathrm{m}$ line is observed by us, we can estimate the contribution from the He I $3 s^{3} S-4 p^{3} P^{o} 1.25 \mu \mathrm{m}$ line. The line ratio of the $\mathrm{He} \mathrm{I} 1.25 \mu \mathrm{m}$ line to the $\mathrm{He} \mathrm{I} 1.7 \mu \mathrm{m}$ line is 0.042 (Robinns 1968), so that the $\mathrm{He}$ I $1.25 \mu \mathrm{m}$ line flux would not be greater than $6 \times 10^{-14} \mathrm{ergs} \mathrm{cm}^{-2} \mathrm{~s}^{-1}$, if we scale the observed He I 1.7 $\mu \mathrm{m}$ line flux to entire nebula using the scaling factor of 9 indicated by the observed H I B10 line. This would have a negligible contribution. In summary, the four most important lines in the $J$ band $\left(\mathrm{H}\right.$ I $\mathrm{Pa} \beta, \mathrm{H}$ I $\mathrm{Pa} \gamma, \mathrm{He}$ II $7-5$, and $\mathrm{He}$ I $\left.2^{3} S-2^{3} P\right)$ are estimated to have expected fluxes $F_{a}=20,9,0$, and 90, respectively $(\mathrm{H} \beta=100)$. Convolving with the normalized transmission function in the $J$ band, their actual contributions to the $J$-band flux are $F_{b}=16,6,0$, and 52 , respectively $(\mathrm{H} \beta=100)$. The total contribution by these lines is thus estimated at 0.74 of the absolute $\mathrm{H} \beta$ flux, or $3.9 \times 10^{-10}$ ergs $\mathrm{cm}^{-2} \mathrm{~s}^{-1}$, which is $\sim 55 \%$ of the total flux in the $J$ band $\left(7.1 \times 10^{-10} \mathrm{ergs} \mathrm{cm}^{-2}\right.$ $\left.\mathrm{s}^{-1}\right)$ and $80 \%$ of the $J$-band excess in-band flux $\left(5.0 \times 10^{-10}\right.$ ergs $\left.\mathrm{cm}^{-2} \mathrm{~s}^{-1}\right)$.

The total line flux in the $H$ band can directly be obtained from the CGS2 spectroscopic measurements. Since the contin- uum is detected in the CGS2 spectrum, it can be used to directly compare with the model continuum. The excess in $H$ band is obtained by subtracting the model continuum from each wavelength point of the CGS2 spectrum (after multiplying it by a factor of 5 for aperture).

In $K$ band, Treffers et al. (1976) derived a theoretical ratio of $I(2.058 \mu \mathrm{m}) / I(\lambda 4471)=0.64$ under Case B. For IC 418 , the $\lambda 4471$ line flux corrected for extinction is measured at 3.47 in units of $\mathrm{H} \beta=100$ (Aller \& Czyzak 1983). The expected He I $2.058 \mu \mathrm{m}$ line flux is, therefore, $F_{a}=2.2$ in units of $\mathrm{H} \beta=100$. Similarly, the expected fluxes of the $\mathrm{H}$ i Br $\gamma$ can be estimated at $F_{a}=2.9$ in units of $\mathrm{H} \beta=100$, using the results of Brocklehurst (1971) and Giles (1977). Correspondingly, the expected contributions after convolving with the $K$-band normalized transmission function are then $F_{b}=2.6$ and $1.4(\mathrm{H} \beta=100)$ for the $\mathrm{H}$ I $\mathrm{Br} \gamma$ and $\mathrm{He}$ I $2.058 \mu \mathrm{m}$ lines, respectively. It results in the contribution of the two lines to the $K$-band flux at 0.04 of the absolute $\mathrm{H} \beta$ flux, i.e., $2.1 \times 10^{-11} \mathrm{ergs} \mathrm{cm}^{-2} \mathrm{~s}^{-1}$. This is about $8 \%$ of the total $K$-band flux $\left(2.7 \times 10^{-10} \mathrm{ergs}^{-2} \mathrm{~s}^{-1}\right)$, or $11 \%$ of the $K$-band excess in-band flux $\left(2.0 \times 10^{-10} \mathrm{ergs} \mathrm{cm}^{-2}\right.$ $\mathrm{s}^{-1}$ ).

The estimated contributions from the above line emissions to the $J$ - and $K$-band fluxes are summarized in Tables 4 and 5, respectively.

While the $J$-band excess emission is dominated by the $\mathrm{He}$ I $1.083 \mu \mathrm{m}$ line, the correction of the near-infrared broad-band fluxes for line emission does not significantly affect the steep slope of the $H$-band continuum. In Figure 5, we have plotted the excess emission before and after correction for line emission in the near-infrared. For comparison, a blackbody with temperature of $1350 \mathrm{~K}$ is also plotted. The existence of hot grains was first suggested by Willner, Becklin, \& Visvanathan (1972). The presence of two dust components, one hot and one cold, is also clearly evident in the infrared spectrum of NGC 7027 (Kwok 1980). The hot dust component may be attributed to nonequilibrium heating of small grains (Sellgren 1984; Phillips, Sanchez, \& Martinez 1984). While the $K$ - and $L$-band excess emission can be explained by radiation from hot dust grains or nonequilibrium heated small grains, the extremely 
TABLE 4

EXPECTED Line FluXes in the $\boldsymbol{J}$ BAND

\begin{tabular}{|c|c|c|c|c|c|c|}
\hline \multirow[b]{2}{*}{ LINES } & \multirow{2}{*}{$\begin{array}{c}\lambda \\
(\mu \mathrm{m})\end{array}$} & \multicolumn{2}{|c|}{$\begin{array}{c}F_{a} \\
(\mathrm{H} \beta=100)\end{array}$} & \multirow{2}{*}{$\begin{array}{l}\text { TRANSMISSION } \\
\text { FUNCTION }^{\mathrm{a}}\end{array}$} & \multicolumn{2}{|c|}{$\begin{array}{c}F_{b} \\
(\mathrm{H} \beta=100)\end{array}$} \\
\hline & & IC 418 & IC 5117 & & IC 418 & IC 5117 \\
\hline $\mathrm{H}_{\mathrm{I}} \mathrm{Pa} \beta$ & 1.282 & 20 & 16 & 0.80 & 16 & 13 \\
\hline H I Pa $\gamma$. & 1.094 & 9 & 9 & 0.64 & 6 & 6 \\
\hline He if $7-5 \ldots \ldots \ldots \ldots \ldots$ & 1.165 & 0. & 0.5 & 0.76 & 0. & 0.4 \\
\hline He I $2^{3} S-2^{3} P$ & 1.083 & 90 & $\ldots$ & 0.58 & 52 & $\ldots$ \\
\hline Total & & & 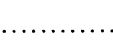 & & 74 & $\ldots$ \\
\hline
\end{tabular}

${ }^{\text {a }}$ Normalized to peak of unity.

TABLE 5

EXPECTED LINE FLUXES IN THE $K$ BAND

\begin{tabular}{|c|c|c|c|c|c|c|}
\hline \multirow[b]{2}{*}{ LINES } & \multirow{2}{*}{$\begin{array}{c}\lambda \\
(\mu \mathrm{m})\end{array}$} & \multicolumn{2}{|c|}{$\begin{array}{c}F_{a} \\
(\mathrm{H} \beta=100)\end{array}$} & \multirow{2}{*}{$\begin{array}{l}\text { TRANSMISSION } \\
\text { FunCTION }\end{array}$} & \multicolumn{2}{|c|}{$\begin{array}{c}F_{b} \\
(\mathbf{H} \beta=100)\end{array}$} \\
\hline & & IC 418 & IC 5117 & & IC 418 & IC 5117 \\
\hline $\mathrm{H}$ I Br $\gamma \ldots$ & 2.166 & 2.9 & 2.7 & 0.91 & 2.6 & 2.5 \\
\hline He I $2^{1} S-2^{1} P$ & 2.058 & 2.2 & 2.8 & 0.65 & 1.4 & 1.8 \\
\hline Total & & & & & 4.0 & 4.3 \\
\hline
\end{tabular}

${ }^{a}$ Normalized to peak of unity.

steep slope seen in the $H$-band CGS2 spectral continuum excess emission cannot be explained by any thermal dust or nebular gas radiation. This rise in the $H$-band excess emission toward the short-wavelength edge of the filter is indicative that a broad feature might be centered at a wavelength between 1.25 and $1.5 \mu \mathrm{m}$.

\subsection{IC 5117}

A model fit to the SED of IC 5117 is presented in Zhang \& Kwok (1991). For comparison, the SEC is reproduced in Figure 6 after incorporating the CGS2 spectrum. Since the angular diameter of this nebula is only 1"4 (Aaquist \& Kwok
1991), there is no correction needed for the aperture size. Besides the near-infrared broad-band $J, H, K$, and $L$ fluxes measured by Kwok et al. (1986), we have also plotted the $J$-, $H$-, $K$-, $L$-, and $M$-band fluxes measured by van der Veen et al. (1989) for comparison. The physical parameters for IC 5117 are also given in Table 2.

The $J$-, $H$-, and $K$-band fluxes of Kwok et al. (1986) are a factor 2-3 lower than those of van der Veen et al. (1989), while the $L$-band fluxes from the two measurements are in good agreement with each other. The cause of these discrepancies is not clear. We note that the $H$-band measurement from Kwok et al. (1986) is located below the flux level of the $H$ band CGS2

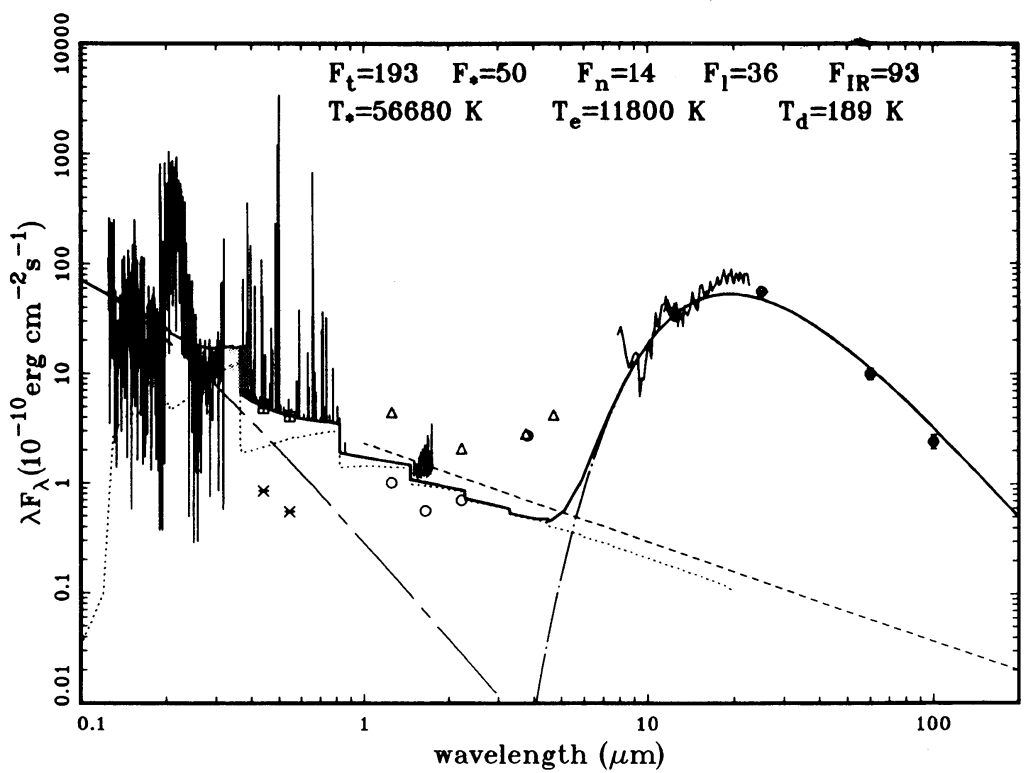

Fig. 6.- The spectral energy distribution of IC 5117. Triangles denote near-infrared $J, H, K, L$, and $M$ band measurements by van der Veen et al. (1989), while open circles are the $J, H, K$, and $L$ band measurements by Kwok et al. (1986). Other symbols used in this diagram are the same as in Fig. 4 for IC 418 . The CGS2 spectrum of this nebula is also shown along with the SED. 
spectrum, whereas the $H$-band flux from van der Veen et al. (1989) is in fair agreement with that of the $H$-band CGS2 spectrum.

The CGS2 spectrum in the $H$ band clearly shows an excess over the sum of the emission from the central star, nebular gas, and the cool dust $(189 \mathrm{~K})$ indicated by the IRAS measurements. The continuum seen in the CGS2 spectrum increases with increasing wavelength and has the same slope as defined by the $H, K$, and $L$ broad-band measurements. After the contributions from the central star, the nebular bound-free and free-free emission, and the cool dust thermal emission $(189 \mathrm{~K}$, indicated by the $I R A S$ measurements) are subtracted, an excess emission in the range $1-4 \mu \mathrm{m}$ is plotted as a function of wavelength in Figure 7.

The spectroscopy of LeVan \& Rudy (1983) shows that the He I $1.083 \mu \mathrm{m}$ line is typically $4-10$ times as strong as $\mathrm{Pa} \beta$ for many planetary nebulae. Since there is no spectroscopic data available for IC 5117, we can only assume that the bulk of the $J$-band excess can be accounted for by the inclusion of this $\mathrm{He}$ I line.

The expected fluxes of the $\mathrm{H}$ I $\mathrm{Br} \gamma$ and $\mathrm{He}$ I $2{ }^{1} S-2{ }^{1} P$ lines can be estimated at $F_{a}=2.7$ and 2.2 using the same procedure for IC 418 above, and $F_{b}=2.5$ and $1.8(\mathrm{H} \beta=100)$, respectively. It results in the contribution of the two lines to the $K$-band flux at $4.3 \%$ of the absolute $\mathrm{H} \beta$ flux, i.e., $3.3 \times 10^{-12}$ ergs $\mathrm{cm}^{-2} \mathrm{~s}^{-1}$, which is about $11 \%$ of the total $K$-band flux $\left(3.1 \times 10^{-10} \mathrm{ergs} \mathrm{cm}^{-2} \mathrm{~s}^{-1}\right)$. The contributions from the line emissions above to the $J$ - and $K$-band fluxes for IC 5117 are also listed in Tables 3 and 4, respectively.

A blackbody curve with a temperature of about $1000 \mathrm{~K}$ is also shown in Figure 7 for comparison. The continuous increase of the excess flux from the $K, L, M$ to the $I R A S 12 \mu \mathrm{m}$ band suggests a temperature gradient in the dust component ranging from 200 to $1000 \mathrm{~K}$.

\section{CONCLUSIONS}

The near-infrared spectra of planetary nebulae IC 418 and IC 5117 in the wavelength range from 1.5 to $1.75 \mu \mathrm{m}$ were obtained and analyzed. Up to 10 members of the hydrogen Brackett series were detected, allowing a comparison of the observed line intensity ratios with the prediction of the recombination line theory. It is found that the Brackett line ratios corrected for the amount of extinction derived from the data in the wavelength range other than near-infrared are in good agreement with the theoretical predictions.

The observed $\mathrm{He}$ I $1.7 \mu \mathrm{m}$ line is found in the two nebulae, and the line flux is in fair agreement with the theory. Some neutral metal emission lines: Mg I $1.504 \mu \mathrm{m}$, Si I 1.583, 1.596, 1.621, and $1.668 \mu \mathrm{m}$ (in IC 5117), and Si I 1.621 and $1.625 \mu \mathrm{m}$ (in IC 418) lines, are present. They must form in a region where these atoms are shielded from being ionized.

The spectral energy distributions of IC 418 and IC 5117 indicate that a significant amount of their energy is emitted in the far-infrared due to thermal emission of dust grains. The central star temperatures of IC 5117 and IC 418 derived from the model fitting to the observed SED are 56,000 and 40,000 K, respectively. This temperature of IC 418 central star is $10 \%$ higher than the effective temperature derived from the analysis of the absorption line profiles of this central star and $5 \%$ higher than the Zanstra temperature derived by Shaw \& Kaler (1989) using the newly determined magnitude of the central star.

One of the important characteristics of the SEDs is that the two nebulae show near-infrared excesses over the underlying radiation from the central star, from the gaseous bound-free, free-free emission, and from the cool dust. Hot dust grains are needed to explain the $H-, K-, L-$, and $M$ - (for IC 5117) band excess. While the majority of the $J$-band excess can be accounted for by the inclusion of the strong He I $1.083 \mu \mathrm{m}$ line emission, the rising of this excess emission seen in the $H$-band CGS2 spectrum of IC 418 toward the short-wavelength end is remarkably mysterious. This slope in the continuum could be due to an unknown dust broad feature positioned at about 1.25-1.5 $\mu \mathrm{m}$. Near-infrared spectroscopy in the bands other than the $H$ band is needed to determine the nature of the excess emission.

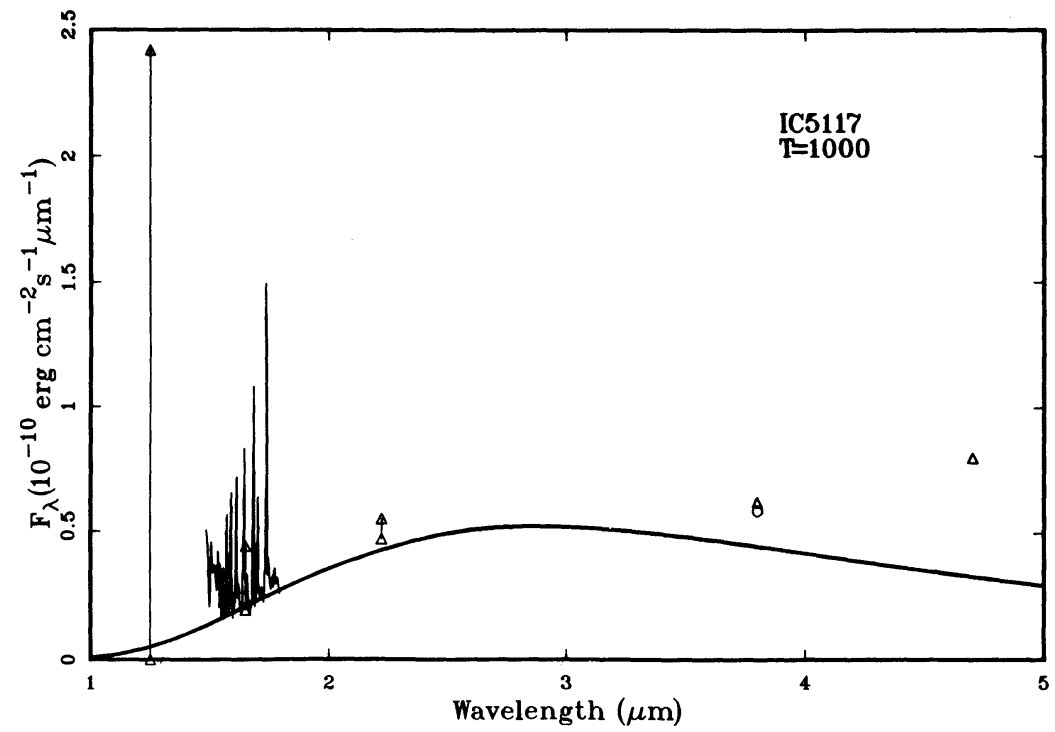

FIG. 7.- The near-infrared excess emission of IC 5117 plotted against wavelengths. Triangles correspond to the measurements of van der Veen et al. (1989), the

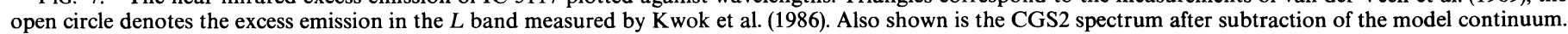
The solid line is a $1000 \mathrm{~K}$ blackbody curve. 
We thank T. R. Geballe and B. J. Hrivnak for assistance during the observations. We also thank S. Torres-Peimbert and H. Dinerstein for helpful discussions. An anonymous referee has made many useful suggestions which we have adopted in the paper. This work is supported by a grant to S. K. from the National Sciences and Engineering Research Council of Canada.

Aaquist, O. B., \& Kwok, S. 1991, ApJ, 378, 599

\section{REFERENCES}

Aitken, D. K., \& Roche, P. F. 1982, MNRAS, 200, 217

Aitken, D. K., Roche, P. F., Spenser, P. M., \& Jones, B. 1979, ApJ, 233,925

Allamandola, L. J., Tielens, A. G. G. M., \& Barker, J. R. 1985, ApJ, 290, L25

Aller, L. H., \& Czyzak, S. J. 1979, ApSS, 62, 397 1983, ApJS, 51, 211

Atlas of Low Resolution IRAS Spectra. 1986, IRAS Science Team, prepared by F. M. Olnon \& E. Raimond (A\&AS, 65, 607)

Balick, B. 1987, AJ, 94, 671

Bashkin, S., \& Stoner, J. D. 1975, Atomic Energy Levels and Grotrian Diagrams, Vol. 1 (Amsterdam: North-Holland)

Brocklehurst, M. 1971, MNRAS, 153, 471

Brown, R. L., \& Mathews, W. G. 1970, ApJ, 160, 939

Cohen, M., Allamandola, L., Tielens, A. G. G. M., Bregman, J., Simpson, J. P., Witteborn, F., Wooden, D., \& Rank, D. 1986, ApJ, 302, 737

Cohen, M., \& Barlow, M. J. 1974, ApJ, 193, 401

Draine, B. T. 1990, in Proc. 22nd Eslab Symposium on Infrared Spectroscopy in Astronomy (ESA SP-290) ed. B. H. Kaldeich (Paris: ESA Publication Division), 93

Forrest, W. J., Houck, J. R., \& McCarthy, J. F. 1981, ApJ, 248, 195

Giles, K. 1977, MNRAS, 180, 57P

Gillett, F. C., Low, F. G., \& Stein, W. A. 1967, ApJ, 149, L97

Higgs, L. A. 1971, Catalog of Radio Observations of Planetary Nebulae and Related Optical Data (Ottawa: National Research Council of Canada)

IRAS Point Source Catalog, Version 2. 1988, Joint IRAS Science Working Group (Washington, DC: GPO)

Kohoutek, L., \& Martin, W. 1981, A\&AS, 44, 325

Kwok, S. 1980, ApJ, 236, 592

Kwok, S., Hrivnak, B. J., \& Milone, E. F. 1986, ApJ, 303, 451

Leger, A., d'Hendecourt, L., \& Defourneau, D. 1989, A\&A, 216, 148

Leger, A., \& Puget, J. L. 1984, A\&A, 137, L5

LeVan, J. P., \& Rudy, R. J. 1983, ApJ, 272, 137

Martin, W. 1987, A\&A, 182, 290

Mendez, R. H. 1989, in IAU Symp. 131, Planetary Nebulae, ed. S. TorresPeimbert (Dordrecht: Kluwer), 261

Mendez, R. H., Kudritzki, R. P., Herrero, A., Husfeld, D., \& Groth, H. G. 1988, A\&A, 190, 113

Mezger, P. G., \& Henderson, A. P. 1967, ApJ, 147, 471

Middlemass, D. 1989, in IAU Symp. 131, Planetary Nebulae, ed. S. TorresPeimbert (Dordrecht: Kluwer), 217

Moseley, H., \& Silverberg, R. F. 1985, in Interrelationships among Circumstellar, Interstellar, and Interplanetary Dust, ed. J. A. Nuth \& R. E. Stencil (NASA CP-2403), 233

Phillips, J. P., Sanchez Magro, C., \& Martinez Roger, C. 1984, A\&A, 133, 395

Pottasch, S. R. 1984, Planetary Nebulae (Dordrecht: Reidel), 294

- 1987, in Late Stages of Stellar Evolution, ed. S. Kwok \& S. R. Pottasch (Dordrecht: Reidel), 355

Pottasch, S. R., Preite-Martinez, A., Olnon, F. M., Mo, J.-E., \& Kingma, S. 1986, A\&A, 161, 363

Preite-Martinez, A., Acker, A., Koppen, J., \& Stenholm, B. 1989, A\&AS, 81, 309

Robinns, R. R. 1968, ApJ, 151, 497

Roche, P. F., \& Aitken, D. K. 1986, MNRAS, 221, 63

Scrimger, J. N. 1984, ApJ, 280, 170

Sellgren, K. 1984, ApJ, 277, 623

Shaw, R. A., \& Kaler, J. B. 1989, ApJS, 69, 495

Thompson, R. I. \& Boroson, T. A. 1977, ApJ, 216, L75

Treffers, R. R., Fink, U., Larson, H. P., \& Gautier, T. N. 1976, ApJ, 209, 793

van der Veen, W. E. C. J., Habing, H. J., \& Geballe, T. R. 1989, A\&A, 226, 108

Volk, K., \& Cohen, M. 1989, AJ, 98, 1918

Whitelock, P. A. 1985, MNRAS, 213, 59

Willner, S. P., Becklin, E. E., \& Visvanathan, N. 1972, ApJ, 175, 699

Willner, S. P., Jones, B., Puetter, R. C., Russell, R. W., \& Soifer, B. T. 1979, ApJ 234, 496

Zhang, C. Y., \& Kwok, S. 1990, A\&A, 237, 479

1991, A\&A, in press 